\title{
Griffin \& West, Kin Discrimination and the Benefit of Helping in Cooperatively Breeding Vertebrates
}

\section{Supplementary information}

\section{Materials and Methods}

We collected relevant literature by: (1) performing literature searches using the ISI Web of Science, with all papers published up to 31 December 2002 considered; (2) forward and backwards searching through the citations of all the papers on our list and other key references (S1); (3) directly contacting researchers working on long-term studies of cooperatively breeding species that were not on our list, to check for the existence of unpublished results. We identified 28 relevant studies 16 on birds and 12 on mammals. Of these, five mammal studies (Belding's ground squirrel (S2), cavy (S3), Japanese macaque (S4), lions (S5), long-tailed macaque(S6)) and one bird study (white-browed sparrow weaver (S7)) were excluded on the basis that they included parentoffspring interactions in their analyses. Of the remaining 22 studies, 18 contained data specifically relating to kin discrimination in offspring care (Table $\mathrm{S} 1$ ). In the majority of cases, data for the calculation of $r_{\text {help }}$ from a species was obtained from the same reference that had provided the data for $r_{\text {kin }}($ Table $S 2)$. In other cases we searched the literature on a species for the relevant data or contacted researchers directly.

Effects sizes $(r)$ were calculated using standard methodology, described in detail elsewhere (S8, S9); see Ref (S10) for a detailed example. Briefly: (a) in some studies the effect size is given, as the correlation coefficient $(r)$, the $\%$ of variance explained $\left(r^{2}\right)$ or the spearman rank correlation coefficient $(r)$; $(b)$ in 
other cases the effect size can be calculated from a test statistic (e.g. $t, \mathrm{~F}, \square^{2}$ or P value) and the sample size. The formulas for calculating $r$ from test statistics are given in standard meta-analyis texts $(\mathrm{S} 8, \mathrm{~S} 9)$, and also implemented in the statistical calculator of the package MetaWin 2.0(S8). 
Table S1 Studies of measures of kin discrimination from which data for meta-analysis were extracted (mean $r_{\text {kin }}$ values and amalgamated $n$ values in bold)

\begin{tabular}{|c|c|c|c|c|c|c|c|c|c|}
\hline Species & Reference & Helping trait & Test statistic & $\begin{array}{l}\text { Effect } \\
\text { size }(r)\end{array}$ & $\begin{array}{l}\text { Sample } \\
\text { size }(n)\end{array}$ & Unit of $n$ & $n$ groups & $\begin{array}{l}\text { Parameter } \\
\text { measured }\end{array}$ & $\bar{n}$ \\
\hline \multicolumn{10}{|l|}{ Mammals } \\
\hline Brown hyaena Hyaena & Owens and Owens & Pup feeding & Calculated from & 0.185 & 159 & Feeding & 1 & Amount & 1 \\
\hline brunnea & 1984(S11) & & paper & & & event & & & \\
\hline Dwarf mongoose Helogale & Creel et al. 1991(S12) & Allosuckling & $\mathrm{F}=15.59, \mathrm{df}=$ & 0.283 & 181 & Dyad & Not given & Probability & \\
\hline parvula & & & 1,179 & & & & & & \\
\hline \multirow[t]{3}{*}{ Lion Panthero leo } & Grinnel et al. 1995(S13) & Defence & $\begin{array}{l}\text { Calculated from } \\
\text { paper }\end{array}$ & 0.224 & 23 & Playback & 20 & Probability & 2 \\
\hline & & & $\begin{array}{l}\text { Calculated from } \\
\text { paper }\end{array}$ & 0.215 & 23 & Playback & 20 & Probability & 2 \\
\hline & & & & 0.219 & 23 & & & & \\
\hline
\end{tabular}




\begin{tabular}{|c|c|c|c|c|c|c|c|c|c|}
\hline \multirow[t]{11}{*}{ Meerkat Suricata suricatta } & Clutton-Brock et al. & Guarding & $\mathrm{P}=0.5$ & 0.255 & 7 & Group & 7 & Amount & \\
\hline & 1999(S14) & & & & & & & & \\
\hline & Clutton-Brock et al. & Pup feeding & $\square^{2}=1.78$ & 0.204 & 43 & Litter & & Amount & \\
\hline & 2001(S15) & & & & & & & & \\
\hline & & & $\mathrm{P}=0.33$ & 0.346 & 15 & Note 4 & $<13$ & Amount & 3 \\
\hline & & & $\mathrm{P}=0.39$ & 0.208 & 17 & Note 4 & $<14$ & Amount & \\
\hline & & & $\mathrm{P}=0.39$ & 0.501 & 6 & Note 4 & $<15$ & Amount & \\
\hline & & & & 0.244 & 43 & & & & \\
\hline & & & Mean from pup & 0.244 & 43 & & & & \\
\hline & & & feeding data & & & & & & \\
\hline & & & only & & & & & & \\
\hline Spotted hyaena Crocuta & Mills 1985(S16) & Foraging & $\mathrm{P}=0.005$ & 0.173 & 262 & Foraging & 1 & Probability & 4 \\
\hline crocuta & & & & & & group & & & \\
\hline \multirow{3}{*}{$\begin{array}{l}\text { Tammar wallaby Macropus } \\
\text { eugenii }\end{array}$} & Blumstein et al. & Aggression & Calculated from & 0.599 & 12 & Expt. pair & 1 & $\mathrm{~N} / \mathrm{A}$ & 5 \\
\hline & $2002(\mathrm{~S} 17)$ & & paper & & & & & & \\
\hline & & & $\mathrm{Rs}=-0.54$ & 0.540 & 12 & Expt. pair & 1 & N/A & \\
\hline
\end{tabular}


Birds

Arabian babbler Turdoides

Wright et al. 1999(S18)

Chick feeding

$\mathrm{P}=0.875$

0.018

74

18

Amount

6

squamiceps

Australian bell miner

Manorina melanophyrus

Australian magpie Gymnorina

tibicen

Florida scrub jay Aphelocoma

c. coerulescens
Clarke 1984(S19)

$\begin{array}{llllll}\mathrm{P}=0.128 & -0.159 & 92 & \text { Note } 7 & 18 & \text { Amount }\end{array}$

$P=0.065$

$\begin{array}{lll}0.152-91 & \text { Note } 7 & 18\end{array}$

$\mathrm{P}=0.065$

$-0.192$

Note 7

18

Amount

$\mathrm{P}=0.59$

$-0.05$

Note 7

18

Amount

Amount

$-\mathbf{0 . 0 5 0} 92$

Chick feeding

Calculated from

$0.540 \quad 7$

Dyad

2

Amount

7

paper

Chick feeding $\quad \square^{2}=0.144$

0.045

72

Helper

12

Probability

2001(S20)

Mumme 1992(S21)

Chick feeding

$=0.02$

$36 \quad$ Helper $\quad 20$ groups Probability
$1987 ; 15$

$1987 ; 15$ 


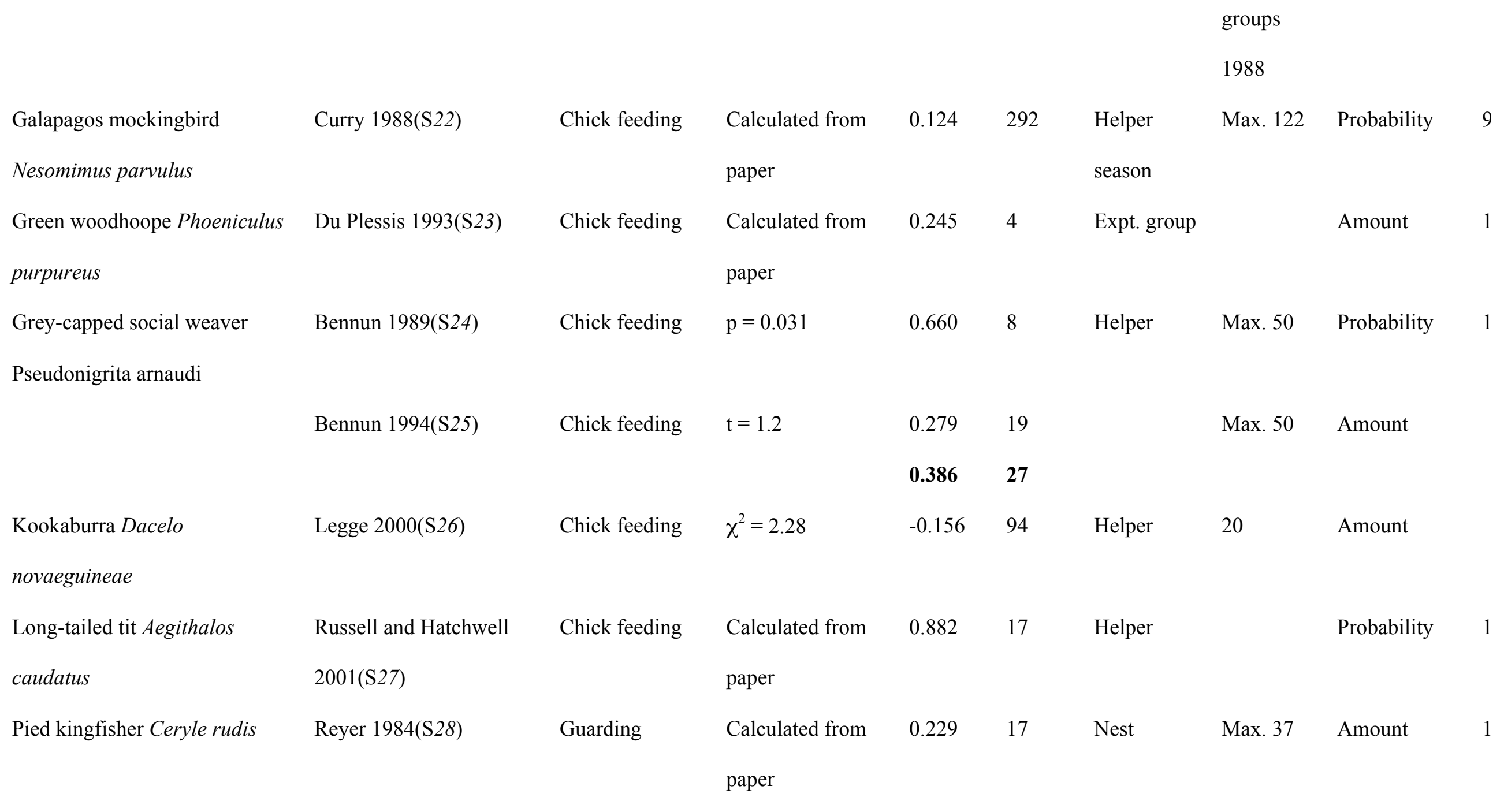




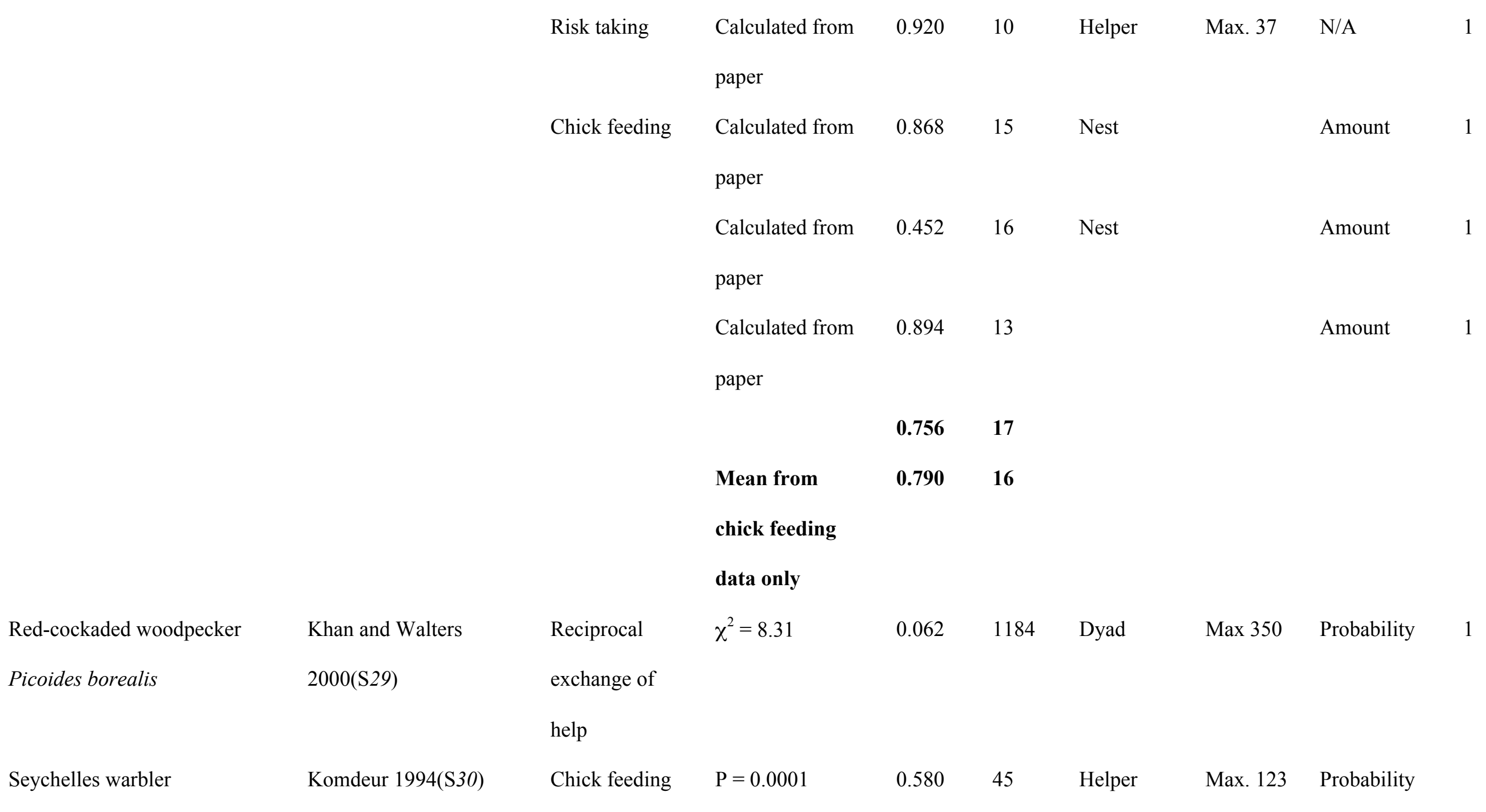


Acrocephalus sechellensis

\begin{tabular}{|c|c|c|c|c|c|c|c|c|}
\hline & & & $\mathrm{P}=0.00003$ & 0.551 & 57 & Helper & Max. 123 & Probability \\
\hline & & & $\mathrm{P}=0.002$ & 0.977 & 10 & Helper & & Probability \\
\hline & & & $\mathrm{T}=4.206$ & 0.903 & 6 & Helper & & Amount \\
\hline & & & $\mathrm{T}=2.496$ & 0.870 & 4 & Helper & & Amount \\
\hline & & & $\mathrm{T}=2.795$ & 0.813 & 6 & Helper & & Amount \\
\hline & & & $\mathrm{T}=2.425$ & 0.864 & 4 & Helper & & Amount \\
\hline & & & $\mathrm{T}=6.190$ & 0.952 & 6 & Helper & & Amount \\
\hline & & & $\mathrm{T}=3.714$ & 0.935 & 4 & Helper & & Amount \\
\hline & & & & 0.815 & 112 & & & \\
\hline Stripe-backed wren & Rabenold 1985(S31) & Chick feeding & Calculated from & -0.208 & 97 & Helper & Max. 30 & Amount \\
\hline Campylorhynchus nuchalis & & & paper & & & & & \\
\hline Superb fairy-wren Malurus & Dunn et al. 1995(S32) & Chick feeding & $\mathrm{F}=1.9, \mathrm{df}=1,21$ & -0.288 & 23 & Helper - & 13 & Amount \\
\hline cyaenus & & & & & & brood dyad & & \\
\hline & & & $r^{2}=0.03$ & 0.173 & 7 & Dyad & & Amount \\
\hline Western bluebird Sialia & Dickinson et al. & Chick feeding & Calculated from & 0.326 & 321 & Helper & 363 & Probability \\
\hline
\end{tabular}


mexicana

White-fronted bee eater

Merops bulockoides
1996(S33)

Emlen and Wrege

1988(S34) paper

Chick feeding $\quad \mathrm{G}=70$

$\begin{array}{lccccl}G=46 & 0.567 & 143 & \text { Dyad } & \text { Not given } & \text { Probability } \\ G=55.1 & 0.521 & 203 & \text { Dyad } & \text { Not given } & \text { Probability } \\ G=41.3 & 0.627 & 105 & \text { Dyad } & \text { Not given } & \text { Probability } \\ \text { Calculated from } & 0.200 & 59 & \text { Dyad } & \text { Not given } & \text { Amount }\end{array}$

paper
0.664 159 Dyad Not given Probability

Legend for Table S1

(1) Re-analysis of data in Table 1 with an ordered heterogeneity test gave $\mathrm{P}=0.01$ (one-tailed), from 159 observations on 24 individuals. (2a) T-test performed on data given in figure $4 \mathrm{~b}$ on proportion of each approach walked in parallel; $t=1.051$. (2b) T-test performed on data given in figure $4 a$ on number of glances made during an approach; $t=1.01$. (3) $n=$ number of comparisons made within sex/age categories, pooled across groups. (4) Analaysis of Kousant group only: we make conservative assumption of $\mathrm{p}=0.05$; P-value given as $<0.05$. We were unable to analyse Kaspersaii group because of 
inconsistency between D-values given and corresponding P-values. (5) Sign test on 10/12 gives $\mathrm{P}=0.019$ (one-tailed). (6) Some birds appear twice in the data set where they were observed to feed two broods in the same nest-site. (7) Correlation on data presented in Table 3 re-done, excluding interactions between direct dscendents. (8) Data presented in Figure 7 re-analysed with ordered heterogeneity test gave $\mathrm{P}=0.0025$. (9a) Ordered regression (df $=1$ ) performed on data on male helpers in Table 4, $\mathrm{G}=2.56$; (9b) on female helpers, $\mathrm{G}=5.64$; (9c) on all helpers, $\mathrm{G}=4.46$. (10) Two-tailed Wilcoxin signed rank test performed on data presented in Figure 1a, $\mathrm{p}=0.625$. (11) Sign test performed on data provided by Bennun gave $\mathrm{P}=0.031, \mathrm{n}=8$. (12) G-test performed on data presented in Figure $4 b, G=26.47$. (13a) Chi-square test performed on data presented in Figure 2a, $T=0.91$; (13b) $r$ calculated from data described in second paragraph of section "Contribution of breeders and helpers to brood care", p1166, $\square^{2}=8.46$; (13c) T-test performed on data presented in Figure 2b, T =6.29; (13d) T-test performed on data presented in Figure 2c, $\mathrm{T}=1.90$; (13e) T-test performed on data presented in Figure 2d, $\mathrm{T}=6.61$. (14) Data presented in Table 3 re-analysed with ordered heterogeneity test gave $\mathrm{P}=0.016$ (one-tailed). (15) Re-analysis of data presented in Figure 4 with an ordered heterogeneity test gave $\mathrm{P}=0.02$ (one-tailed). (16) Re-analysis of raw data in Table 4 gave $\square_{(1)}^{2}=334.15$. (17) $r$ calculated from statement on p311 "Genetic relatedness explained only $4 \%$ of the total variance in helper feeding rate..." 
Table S2 Studies of measures of the effect of helpers, from which data for meta-analysis were extracted.

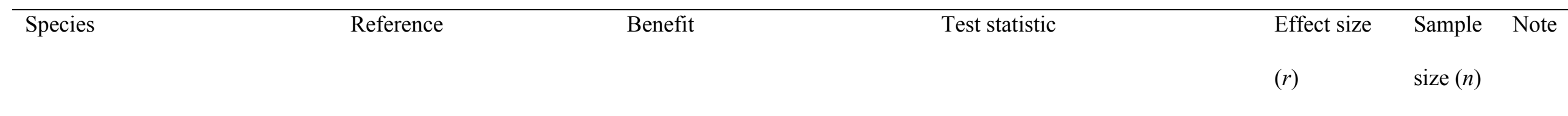

Mammals

Dwarf mongoose Helogale

Creel et al. 1991(S12)

Litter size

$\mathrm{t}=3.58$

0.656

19

parvula

Meerkat Suricata suricatta

Russell pers.comm.;

Survival to 1 year

$\mathrm{F}=15.91$;

0.323

139

Clutton-Brock et al.

$\mathrm{df}=1,137$

2001(S35)

\section{Birds}

Arabian babbler Turdoides

Wright et al. 1998(S36)

Fledgling surviving to

$r^{2}=0.24$

0.490

27

squamiceps

independence 
tibicen

Florida scrub jay Aphelocoma c.

coerulescens

tailed)

Green woodhoopoe

Du Plessis 1993(S23)

Number of fledglings

Calculated from paper

0.1018

Phooeniculus pupureus

Kookaburra Dacelo

novaeguineae

Pied kingfisher Ceryle rudis

tailed)

Seychelles warbler

Acrocephalus sechellensis

Stripe-backed wren

Rabenold 1984(S39) Nunmber of juveniles

$\mathrm{t}=3.182$

0.662

Campylorynchus nuchalis

Superb fairy-wren Malarus

Dunn et al. 1995(S32)

Young surviving to 4 weeks

cyaenus

Western bluebird Sialia

Dickinson et al. 1996(S33) Chance of raising at least one 
mexicana

offspring

White-fronted bee eater Merops

Emlen and Wrege

Number of fledglings

$\mathrm{r}^{2}=0.35$

0.592

bulockoides

1988(S34)

Legend for Table S2.

(1) Data presented in Table 3 analysed to give $t=1.22$. (2) Data presented in Table 6 analysed to give $t=6.94$. (3) Regression performed on data presented in Figure 5. 
S1. S. T. Emlen, in Behavioral Ecology J. R. Krebs, N. B. Davies, Eds. (Blackwell, Oxford, UK, 1997) pp. $228-253$.

S2. P. Sherman, Science 197, 1246-1253 (1977).

S3. J. Kunkele, H. Hoeck, Behavioral Ecology and Sociobiology 37, 385-391 (1995).

S4. B. Chapais, L. Savard, C. Gauthier, Behavioral Ecology and Sociobiology 49, 493-502 (2001).

S5. A. E. Pusey, C. Packer, Behavioral Ecology 5, 362-374 (1994).

S6. H. Schaub, International Journal of Primatology 17, 445-467 (1996).

S7. R. Magrath, L. Whittingham, Behavioral Ecology and Sociobiology 41, 185-192 (1997).

S8. M. S. Rosenberg, D. C. Adams, J. Gurevitch. (Sinauer, Sunderland, Massachusetts, 2000).

S9. R. Rosenthal, Meta-analytic procedures for social research. (Sage Foundation, California, 1991).

S10. M. D. Jennions, A. P. Moller, M. Petrie, Quarterly Review of Biology 76, 3-36 (2001).

S11. D. Owens, M. Owens, Nature 308, 843-845 (1984).

S12. S. Creel, S. Monfort, D. Wildt, P. M. Waser, Nature 351, 660-662 (1991).

S13. J. Grinnell, C. Packer, A. E. Pusey, Animal Behaviour 49, 95-105 (1995).

S14. T. H. Clutton-Brock et al., Science 284, 1577-1724 (1999).

S15. T. H. Clutton-Brock et al., Science 291, 478-481 (2001).

S16. M. Mills, Nature 316, 61-62 (1985).

S17. D. Blumstein, J. G. Ardon, C. S. Evans, Ethology 108, 815-823 (2002).

S18. J. Wright, P. Parker, K. Lundy, Animal Behaviour 58, 779-785 (1999).

S19. M. Clarke, Behavioral Ecology and Sociobiology 14, 137-146 (1984).

S20. P. Finn, J. Hughes, Emu 101, 57-63 (2001).

S21. R. L. Mumme, Behavioral Ecology and Sociobiology 31, 319-328 (1992).

S22. R. Curry, Behavioral Ecology and Sociobiology 22, 141-152 (1988).

S23. M. A. Du Plessis, Behaviour 127, 49-65 (1993).

S24. L. Bennun, Oxford University (1989).

S25. L. Bennun, Animal Behaviour 47, 1047-1056 (1994).

S26. S. Legge, Animal Behaviour 59, 1009-1018 (2000). 
S27. A. F. Russell, B. J. Hatchwell, Proceedings of the Royal Society of London Series B-Biological Sciences 268, 2169-2174 (2001).

S28. H. Reyer, Animal Behaviour 32, 1163-1178 (1984).

S29. M. Khan, J. Walters, Behavioral Ecology and Sociobiology 47, 376-381 (2000).

S30. J. Komdeur, Proceedings of the Royal Society of London Series B-Biological Sciences 256, 47-52 (1994).

S31. K. N. Rabenold, Behavioral Ecology and Sociobiology 17, 1-17 (1985).

S32. P. O. Dunn, A. Cockburn, R. A. Mulder, Proceedings of the Royal Society of London Series B-Biological Sciences 259, 339-343 (1995).

S33. J. Dickinson, W. D. Koenig, F. Pitelka, Behavioral Ecology 7, 168-177 (1996).

S34. S. T. Emlen, P. Wrege, Behavioral Ecology and Sociobiology 23, 305-315 (1988).

S35. T. H. Clutton-Brock et al., Science 293, 2446-2449 (2001).

S36. J. Wright, Journal of Avian Biology 29, 105-112 (1998).

S37. S. Legge, Journal of Animal Ecology 69, 714-724 (2000).

S38. J. Komdeur, Behavioral Ecology and Sociobiology 34, 175-186 (1994).

S39. K. N. Rabenold, Ecology 65, 871-885 (1984).

\section{Supporting Online Material}

www.sciencemag.org

Materials and Methods

Tables S1, S2 